\title{
HuR is exported to the cytoplasm in oral cancer cells in a different manner from that of normal cells
}

\author{
H Hasegawa ${ }^{1,2,4}$, W Kakuguchi 1,2,4, T Kuroshima',3, T Kitamura', S Tanaka',2, Y Kitagawa ${ }^{3}$, Y Totsuka ${ }^{2}$, \\ M Shindoh' and F Higashino*,I
}

'Department of Oral Pathology and Biology, Hokkaido University Graduate School of Dental Medicine, North 13 West 7, Kita-ku, Sapporo 060-8586, Japan; ${ }^{2}$ Department of Oral and Maxillofacial Surgery, Hokkaido University Graduate School of Dental Medicine, North 13 West 7, Kita-ku, Sapporo 060-8586, Japan; 'Department of Oral Diagnosis and Oral Medicine, Hokkaido University Graduate School of Dental Medicine, North 13 West 7.

Kita-ku, Sapporo 060-8586, Japan

\begin{abstract}
HuR, a ubiquitously expressed member of the Hu protein family that binds and stabilizes an AU-rich element (ARE)-containing mRNAs, is known to shuttle between the nucleus and the cytoplasm via several export pathways. When normal cells were treated with heat shock, HuR was exported to the cytoplasm in a chromosome maintenance region I (CRMI)-dependent manner. However, in this study, we demonstrate that HuR is exported to the cytoplasm in oral cancer cells even if the cells were treated with the inhibitor of the CRMI-independent export pathway. Immunohistochemical and biochemical analyses showed that HuR existed in both the cytoplasm and the nucleus in oral cancer cells, such as HSC-3 and Ca9.22, but existed entirely inside the nucleus in normal cells. AU-rich element-mRNAs were also exported to the cytoplasm and stabilised in the oral cancer cells, which were inhibited by HuR knockdown. This export of HuR was not affected by at least $7 \mathrm{~h}$ of treatment of leptomycin B (LMB), which is an inhibitor of the CRMI-dependent export pathway. These findings suggest that HuR is exported to the cytoplasm in oral carcinoma cells in a different manner from that of normal cells, and is likely to occur through the perturbation of a normal export pathway.

British Journal of Cancer (2009) I 00, 1943-1948; doi:I0.1038/sj.bjc.6605084 www.bjcancer.com

(c) 2009 Cancer Research UK
\end{abstract}

Keywords: HuR; export; AU-rich element; CRMI; oral cancer

HuR is a member of the ELAV (embryonic lethal abnormal vision) family of RNA-binding proteins, which has three RNA recognition motifs connected by a short hinge region (Ma et al, 1996). Although HuR is localised predominantly in the nucleus, it is able to shuttle between the nucleus and the cytoplasm. The export of HuR is mediated by its association with transportin $1(\operatorname{Trn} 1)$ and Trn2 (Rebane et al, 2004) by the shuttling sequence termed 'HNS' in the hinge region (Fan and Steitz, 1998), and by its association with pp32 and APRIL, which includes the nuclear export signal recognised by the export receptor chromosome maintenance region 1 (CRM1) (Brennan et al, 2000; Gallouzi et al, 2001). Under physiological conditions, such as heat shock or serum stimulation, the HuR - pp32 complex is used for the transportation of AU-rich element (ARE)-containing mRNAs from the nucleus to the cytoplasm (Brennan et al, 2000; Gallouzi and Steitz, 2001).

An ARE is commonly present in the $3^{\prime}$-untranslated region of many proto-oncogenes, growth factors and cytokine mRNAs (Chen and Shyu, 1995; Jacobson and Peltz, 1996). Multiple copies of the sequence, AUUUA, often exist in the ARE and they target ARE-mRNAs for rapid degradation (Chen and Shyu, 1995; Brennan and Steitz, 2001). Numerous proteins are known to interact with AREs and modulate either the stabilisation or destabilisation of ARE-mRNAs (Chen and Shyu, 1995; Jacobson and Peltz, 1996; Antic and Keene, 1997; Brennan and Steitz, 2001).

\footnotetext{
*Correspondence: Dr F Higashino; E-mail: fhigashi@den.hokudai.ac.jp

${ }^{4}$ These authors contributed equally to this work

Received 21 November 2008; revised 6 March 2009; accepted 8 April 2009
}

Among such proteins, HuR binds to AREs to protect ARE-mRNAs from rapid degradation (Brennan and Steitz, 2001).

In the cells transformed by an adenovirus oncogene product, ARE-mRNAs and their associated proteins, such as HuR and pp32, are exported to the cytoplasm in a CRM1-independent manner (Higashino et al, 2005). Therefore it is interesting to investigate the export pathway of ARE-mRNA and its binding partner proteins in non-virus-mediated tumour cells.

In this study, we provide evidence regarding the export of $\mathrm{HuR}$ to the cytoplasm in oral cancer cells. We found that ARE-mRNA was also simultaneously exported and accumulated in these cells and that leptomycin B (LMB) treatment failed to inhibit the HuR export. These data indicate that HuR and ARE-mRNAs are exported to the cytoplasm in oral cancer cells in a manner different from that of normal cells. In addition, these findings indicate that this HuR export can be used as a diagnostic marker for oral cancers.

\section{MATERIALS AND METHODS}

\section{Cells and cell fractionation}

Human oral cancer cells, HSC-3 (a human tongue squamous cell carcinoma cell line) and Ca9.22 (a human gingival squamous cell carcinoma cell line), and human oral normal cells, human gingival fibroblast (HGF) and periodontal ligament (PDL) cells, were cultured at $37^{\circ} \mathrm{C}$ with $5 \% \mathrm{CO}_{2}$ in DMEM (Nissui Seiyaku, Tokyo, Japan) containing $10 \%$ foetal bovine serum with penicillin/ streptomycin (Sigma, St Louis, MO, USA). Some of these cells 
were treated with heat shock $\left(45^{\circ} \mathrm{C}, 1 \mathrm{~h}\right)$ and some were treated with LMB (Sigma) $\left(5 \mathrm{ng} \mathrm{ml}^{-1}\right)$ for $7 \mathrm{~h}$.

Cell fractionation was carried out by separating the cells into cytoplasmic and nuclear fractions, as previously described (Weigel and Dobbelstein, 2000), by harvesting and re-suspending the cells in a fractionating buffer (10 mM Tris-HCl, pH 7.6; $150 \mathrm{~mm} \mathrm{NaCl} ; 1.5 \mathrm{~mm}$ $\mathrm{MgCl}_{2}$ and $0.5 \%$ Nonidet P-40 (Sigma-Aldrich, St Louis, MO, USA), protease inhibitor cocktail), followed by vigorous shaking for $5 \mathrm{~min}$ and centrifugation at 12000 r.p.m. for 30 s. The supernatant was used as the cytoplasmic fraction. To estimate the accuracy of cell fractionation, cytoplasmic protein, $\beta$-tubulin (Santa Cruz Biotechnology, Santa Cruz, CA, USA), and nuclear protein, Lamin A/C (Santa Cruz), were detected by immunoblotting.

\section{Immunohistochemical and immunofluorescence analyses}

Tissue samples were obtained from excised tongue carcinomas of patients at the Hokkaido University Dental Hospital, Hokkaido, Japan, and from normal tongue tissues. Informed consent was obtained from the patients before the samples were used. Immunohistochemical detection of HuR was carried out using the avidinbiotin complex method as previously described (Shindoh et al, 1996).

Cells were fixed with $4 \%$ formaldehyde, permeabilised with $0.1 \%$ Triton X-100 and incubated with antibodies specific to HuR (Santa Cruz). After incubation with HuR-specific antibodies, the cells were again incubated with FITC-conjugated secondary antibodies (Molecular Probes, Carlsbad, CA, USA). The cells were observed using an OLYMPUS IX71 (Olympus, Tokyo, Japan) fluorescence microscope.

\section{Western blot analysis}

Western blot analysis was performed using antibodies specific to HuR (Santa Cruz), $\beta$-tubulin, Lamin A/C, CRM1 (BD Biosciences, San Jose, CA, USA) and $\beta$-actin (Sigma), as previously described (Aoyagi et al, 2003).

\section{Quantitative real-time RT - PCR}

To determine the quantity of ARE-mRNAs, the cells were treated with TRI REAGENT (Sigma), after which their RNA was subjected to reverse transcription using Rever Tra Ace (TOYOBO, Osaka, Japan). For quantitative real-time RT - PCR analysis, amplification was performed in a DNA Engine-Opticon 2 PCR machine (M) Research, Waltham, MA, USA) with SYBR Green PCR master mix (DyNAmo SYBR Green qPCR Kit, MJ Research) as previously described (Higashino et al, 2005).

To evaluate the half-life of $c$ - $m y c$ mRNA, HSC-3 and HGF cells were treated with actinomycin D (Act.D) (Sigma) $\left(5 \mu \mathrm{g} \mathrm{ml}^{-1}\right)$ for 30 or $60 \mathrm{~min}$. The extracted RNA was subjected to quantitative real-time RT-PCR.

\section{In situ hybridisation}

In situ hybridisation was performed according to a previously described method (Higashino et al, 2005). The cells were fixed in cold $3 \%$ formaldehyde and permeabilised with cold $0.5 \%$ Triton $\mathrm{X}-100$. Hybridisation was performed overnight at $37^{\circ} \mathrm{C}$ in $20 \mu \mathrm{l}$ of a mixture containing $2.5 \mu \mathrm{g}$ of tRNA, $10 \mu \mathrm{g}$ of salmon sperm DNA, $2 \times$ SSC, $0.2 \%$ BSA, $1 \mathrm{~mm}$ vanadyl ribonucleoside complexes, $50 \%$ formamide, $10 \%$ dextran sulphate and 10-30 ng of 3'-digoxigenin (DIG)-labelled anti-sense deoxyoligonucleotide probe (Hokkaido System Science, Sapporo, Hokkaido, Japan) for $c$-fos or $c-m y c$ mRNA. The coverslips were washed thrice with $2 \times$ SSC (Invitrogen, Carlsbad, CA, USA) at $37^{\circ} \mathrm{C}$ and thereafter with $1 \times \mathrm{SSC}$ at room temperature. After washing, they were incubated for $60 \mathrm{~min}$ at room temperature with a dilution of $1: 50$ of anti-DIG fluorescein Fab fragments (Roche, Basel, Switzerland) in 0.2\% Triton X-100/
PBS containing 1\% BSA (Sigma). After incubation, the coverslips were washed twice with $0.2 \%$ Triton X-100/PBS and thereafter with only PBS. The probes (sense and anti-sense) used were complementary to the nucleotides $288-328$ of $c$-fos and to the nucleotides $6278-6311$ of $c-m y c$. Nuclei of the cells were stained using DAPI (4',6'diamidino-2-phenylindole) (Roche).

\section{HuR knockdown}

To knockdown HuR, siRNA was transfected using RNAiMAX (Invitrogen) as per the manufacturer's instructions. The knockdown level of HuR mRNA was analysed by western blot analysis. HuR siRNA was $5^{\prime}$-uuaccaguuucaauggucatt- $3^{\prime}$, and the control siRNA was Silencer Negative Control \#1 siRNA (Invitrogen).

\section{RESULTS}

\section{Localisation of HuR in oral cancer cells}

To determine the subcellular localisation of HuR in oral cancer cells, we carried out an immunohistochemical analysis of HuR using oral cancer cells, HSC-3 (tongue carcinoma) and Ca9.22 (gingival carcinoma) cells, and normal oral cells, HGF and PDL cells. In HSC-3 and Ca9.22 cells, both the cytoplasm and the nucleus were positively stained by HuR antibody, whereas in HGF and PDL cells, only the nucleus was stained (Figure 1A). These data suggest that $\mathrm{HuR}$ is localised in the nucleus and cytoplasm of the oral cancer cells, whereas almost all of it is localised in the nucleus of normal cells.

Thereafter, we stained the oral cancer tissue (tongue carcinoma) with the same antibody, which showed a cytoplasmic staining of $\mathrm{HuR}$ (Figure 1B, arrows) as against the nuclear staining observed in the adjacent normal cells. In contrast, HuR was localised in the nucleus in normal tissues (Figure 1B). These data indicate the fact that HuR is present in the cytoplasm of oral cancer cells.

To confirm the cytoplasmic localisation of HuR, the cells were separated into cytoplasmic and nuclear fractions, and the HuR of each fraction was detected by western blotting. The amounts of $\mathrm{HuR}$ in the cytoplasm of HSC-3 and Ca9.22 cells were much higher than those observed in the cytoplasm of normal cells (Figure 1C). These results also suggest that HuR is localised in the cytoplasm of oral cancer cells.

Together, these results indicate that HuR is present in the nucleus of normal cells as well as in both the nucleus and the cytoplasm of oral cancer cells, which further suggest that HuR is exported to the cytoplasm in oral cancer cells.

\section{Export and stabilisation of ARE-mRNAs in oral cancer cells}

We examined the export of ARE-mRNA in oral cancer cells, as AREmRNA is known to be exported to the cytoplasm with its binding partner HuR (Brennan et al, 2000; Higashino et al, 2005). The subcellular localisation of $c$-fos and $c$-myc mRNAs in oral cancer cells (HSC-3 and Ca9.22) and in normal cells (HGF) was confirmed by in situ hybridisation. These mRNAs were detected in the nucleus and cytoplasm of HSC-3 and Ca9.22 cells, but were localised only in the nucleus of HGF cells (Figure 2A). These data suggest the export of ARE-mRNAs to the cytoplasm in oral cancer cells.

It has been previously reported that the exported ARE-mRNA is stabilised in the cells transformed with adenovirus E4orf6 (Higashino et al, 2005). In this study, we also examined the stabilisation of ARE-mRNA in oral cancer cells. The amount of $c$ - $m y c$ mRNA expressed in oral cancer (HSC-3 and Ca9.22) and normal (HGF) cells was measured by quantitative real-time RT-PCR. Accumulation of the ARE-mRNAs was greater in the HSC-3 and Ca9.22 oral cancer cells than in the normal cells 


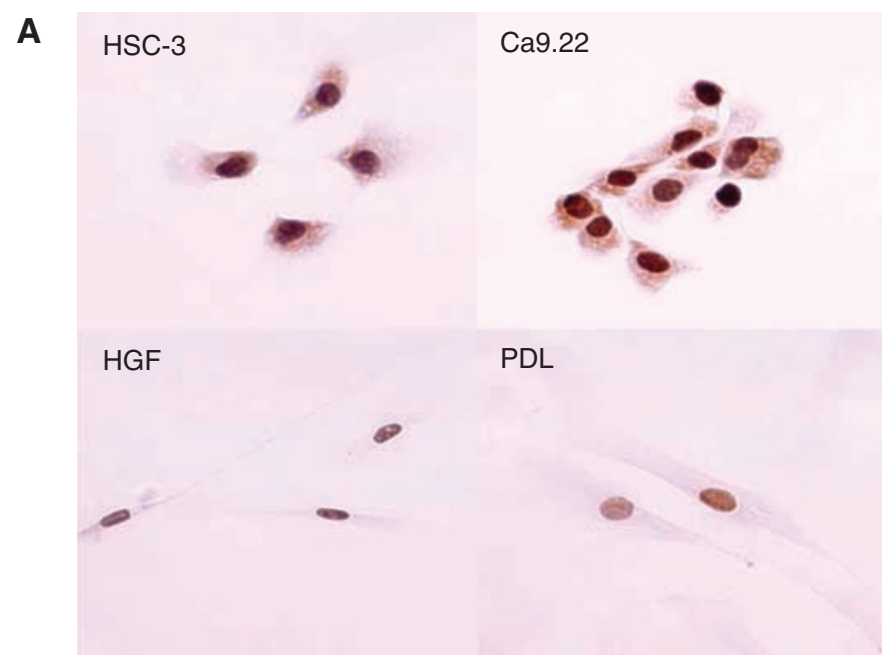

B
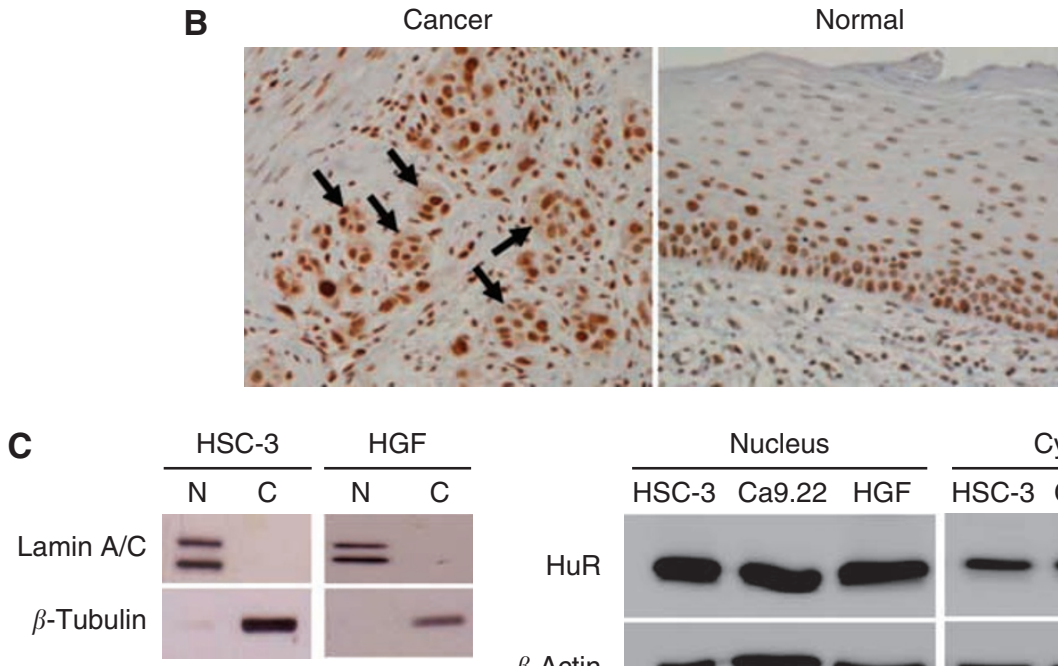

$\mathrm{N}$ : Nucleus

C: cytoplasm

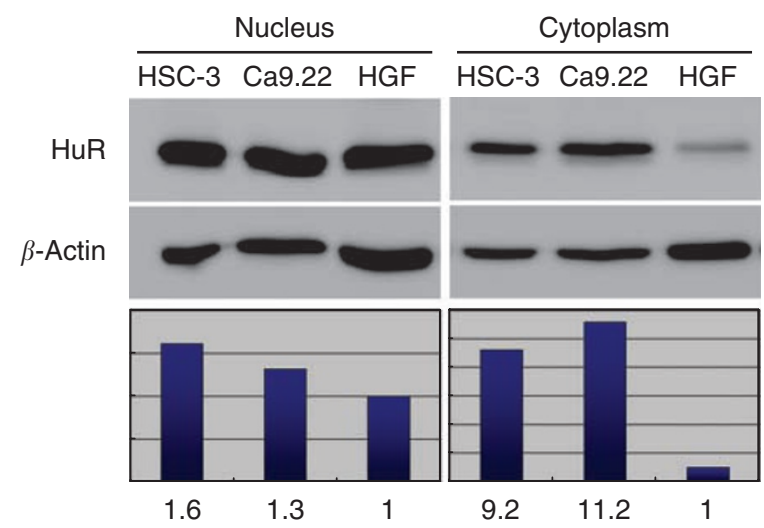

Figure I Subcellular localisation of HuR in oral cancer cells. (A) Immunohistochemical detection of HuR in oral cancer (HSC-3 and Ca9.22) and normal (HGF and PDL) cells. (B) Immunohistochemical detection of HuR in human oral cancer (tongue carcinoma, arrows) and normal oral tissues. Representative photomicrographs of HuR expression in both tissues are shown at $\times 200$ magnification. (C) Western blot analysis of the nuclear and cytoplasmic fractions of HSC-3, Ca9.22 and HGF cells using HuR and $\beta$-actin antibodies. The fractions were analysed by antibodies against $\beta$-tubulin and Lamin A/C. Quantitative assessment of HuR protein in each fraction is shown as a histogram. Hold changes in the HuR level as measured by densitometry is shown.

(Figure 2B). Moreover, to compare the half-life of $c$-myc mRNA, HSC-3 and HGF cells were treated with Act.D, and then the quantity of $c-m y c$ mRNA was measured by real-time RT - PCR. The half-life of the mRNA in HSC-3 cells was longer than that of HGF cells (Figure 2B). These results suggest the stabilisation of AREmRNA in oral cancer cells.

To explore the role of $\mathrm{HuR}$ for the export and stabilisation of ARE-mRNA in cancer cells, HSC-3 cells were subjected to HuR knockdown. In HuR-knockdown cells, $c-m y c$ mRNA was in the nucleus or in the perinuclear region, although the mRNA existed in both the cytoplasm and the nucleus (Figure 2C). In addition, the quantity of $c-m y c$ mRNA decreased in the HuR-knockdown cells compared with that in the cells transfected with the control siRNA (Figure 2C). These results indicate that the export and the increased accumulation of $c-m y c$ mRNA are indeed because of HuR in oral cancer cells.

\section{Export of HuR in the presence of LMB}

HuR is known to be exported to the cytoplasm in a manner dependent on CRM1, which is a member of the exportin family of nuclear transporters, when cells are stimulated by heat shock or serum stimulation (Brennan et al, 2000; Gallouzi and Steitz, 2001). On the other hand, HuR and ARE-mRNAs are known to be exported to the cytoplasm through a CRM1-independent pathway in cells transformed by an adenovirus oncogene product (Higashino et al, 2005). To study the export pathway of HuR in oral cancer cells, an immunofluorescence analysis of the cancer 
A

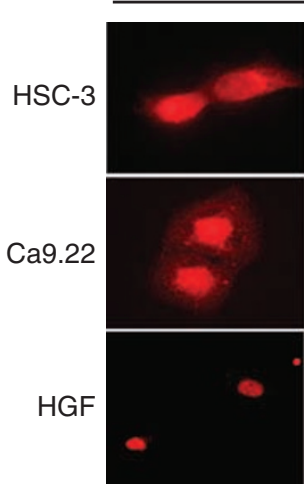

c-fos (sense)
DAPI

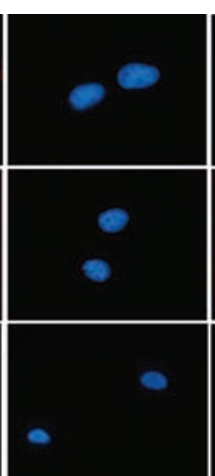

DAPI

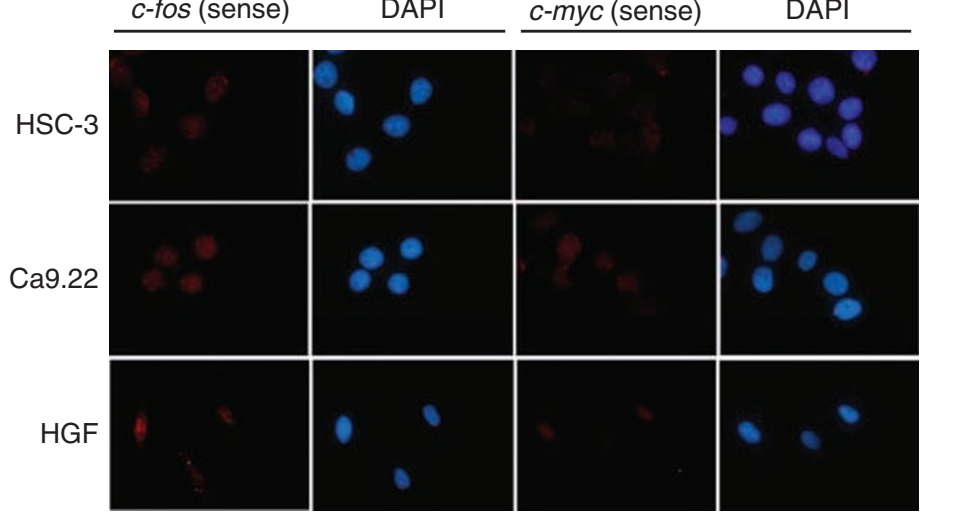

$c-m y c$

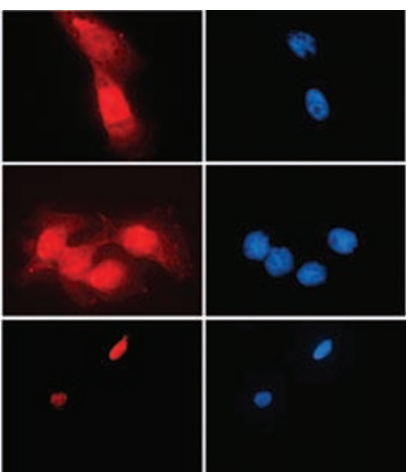

$c-m y c$ (sense)

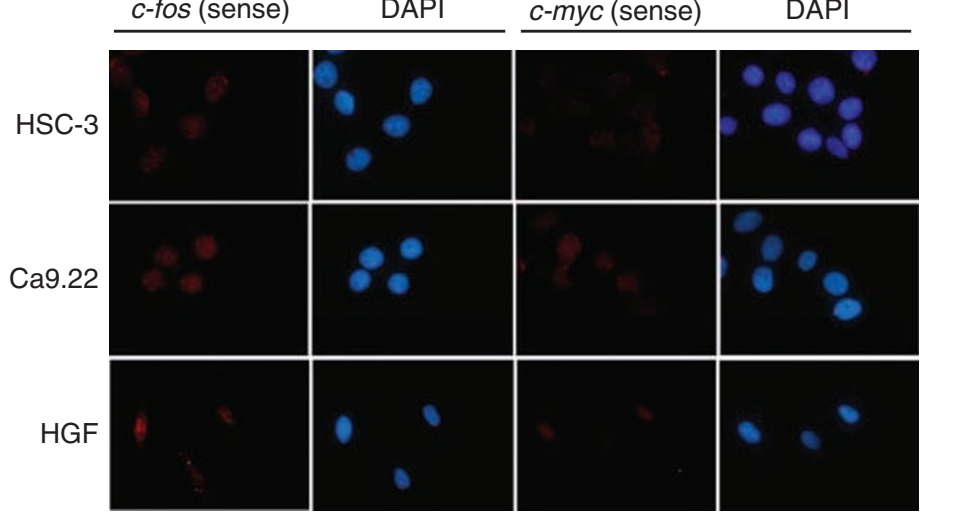

B

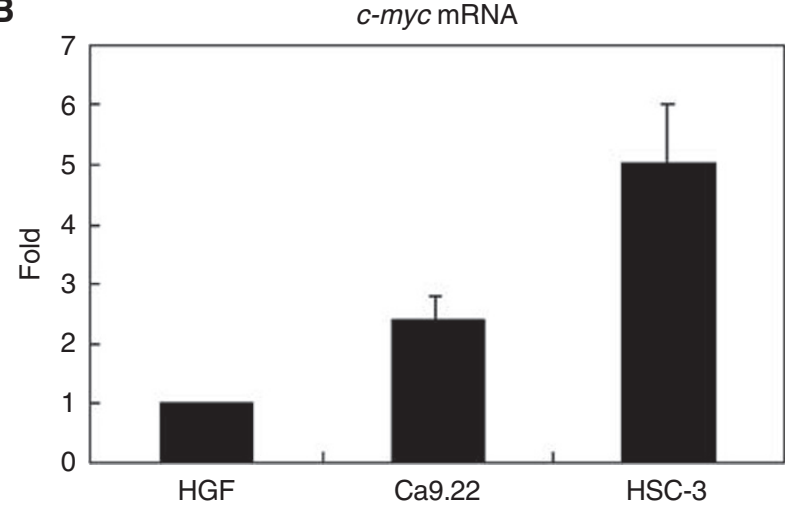

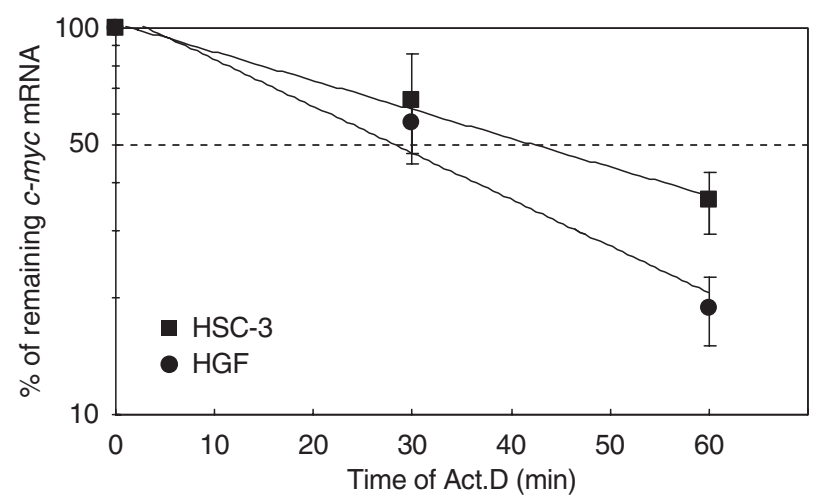
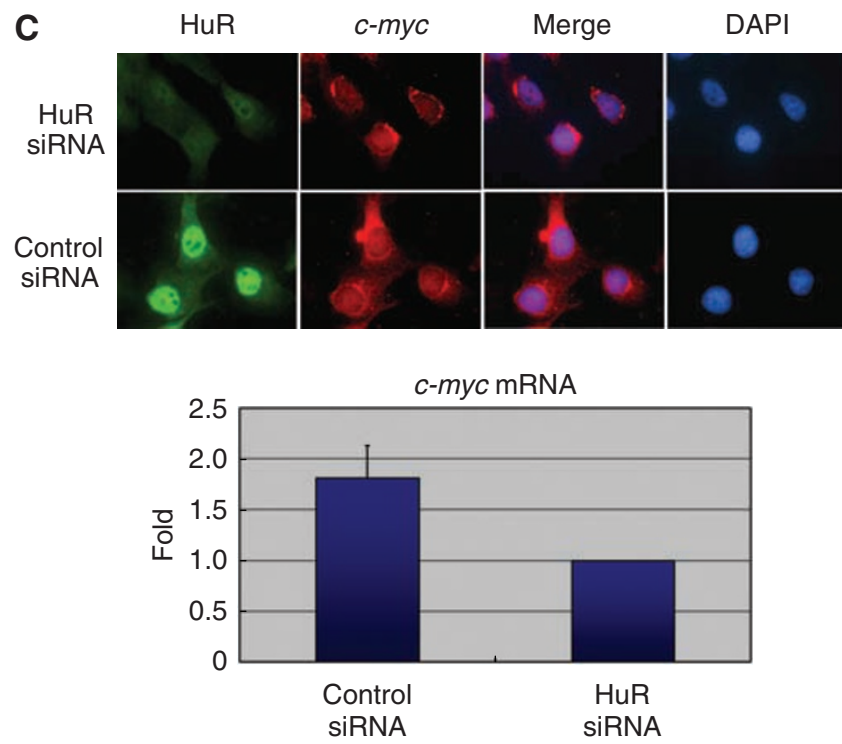

Figure 2 Export and stabilisation of ARE-mRNAs in oral cancer cells. (A) The distribution of c-fos and c-myc mRNAs in HSC-3, Ca9.22 and HGF were detected by in situ hybridisation using digoxigenin-labelled anti-sense (upper) and sense (lower) probes complementary to c-fos and c-myc mRNAs. The DAPI-stained nuclei are shown. (B) The accumulation of c-myc mRNAs expressed in each cell was measured by quantitative real-time RT-PCR (upper) Each cell was treated with Act.D and the amount of c-myc mRNA was estimated at the indicated time by quantitative real-time RT-PCR (lower). Data are mean \pm s.e.m. of three independent experiments. (C) The localisation (upper) and accumulation (lower) of c-myc mRNA in HuR-knockdown HSC-3 cells.

cells, to examine the localisation of HuR, was carried out after their treatment with LMB, an inhibitor of CRM1. In normal cells, heat shock treatment induced stress granules (SGs), which include HuR, and LMB treatment reduced HuR-included SGs (Figure 3A). Conversely, the accumulation of HuR to the cytoplasm in the oral cancer cells was not inhibited, even after the cells were treated with LMB for $7 \mathrm{~h}$ (Figure 3A).
To further confirm the effect of LMB, the existence of HuR in the cytoplasm was examined by western blotting. In normal HGF cells, HuR was observed in the cytoplasm after heat shock treatment $\left(45^{\circ} \mathrm{C}, 1 \mathrm{~h}\right)$, but the HuR band was not visible in the lysate of the cells treated with LMB before the heat shock. On the other hand, in HSC-3 cells, HuR was observed in the cytoplasm even after the cells were treated with LMB (Figure 3B). Together, these data 
A

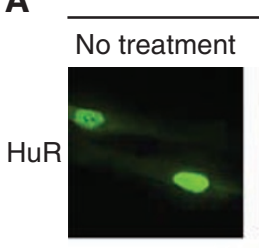

\section{HS}

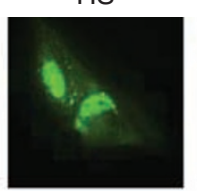

HGF

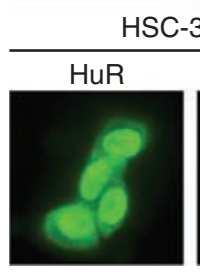

HSC-3 LMB(+)
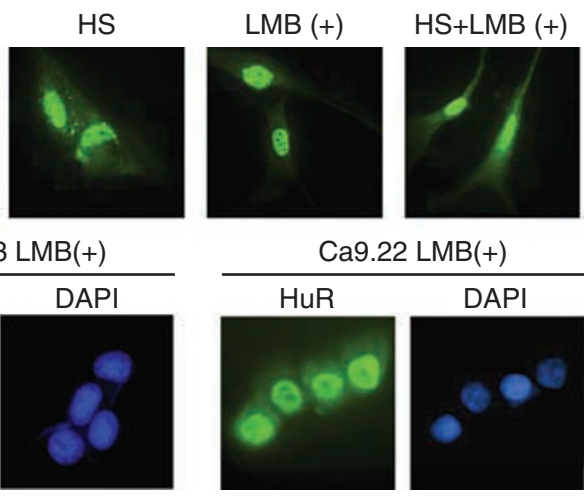

B

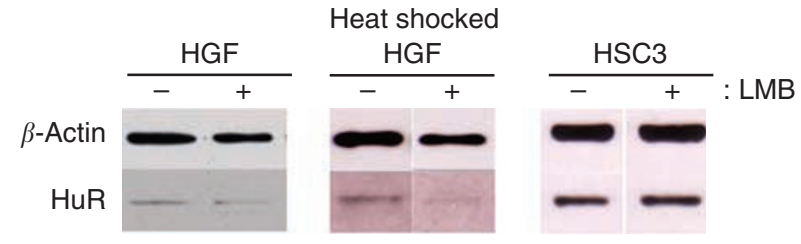

Figure 3 Role of CRMI in the export of HuR in oral cancer cells. (A) The effect of LMB on the export of HuR was observed by an immunofluorescence analysis using HuR antibody. The HGF cells treated with heat shock in the presence and absence of LMB are shown (upper). HuR localisation in the LMB-treated HSC-3 and Ca9.22 cells, stained with DAPI are shown. (lower) (B) The same cells used in panel A were separated into nuclear and cytoplasmic fractions, and the amount of cytoplasmic HuR was confirmed by western blotting.

indicate that, in oral cancer cells, the quantity of HuR in the cytoplasms appeared to be unchanged at least $7 \mathrm{~h}$ treatment of $\mathrm{LMB}$, and that HuR is exported to the cytoplasm by perturbing the physiological CRM-dependent export machinery.

\section{DISCUSSION}

This study shows that HuR and ARE-mRNAs are exported to the cytoplasm in oral cancer cells, and that ARE-mRNA accumulates in these cells. In addition, this study also shows that LMB fails to inhibit the accumulation of HuR in the cytoplasm of oral cancer cells. These results indicate that $\mathrm{HuR}$ and ARE-mRNAs are exported from the nucleus to the cytoplasm in oral cancer cells in a manner different from that of normal cells, and that this HuR export can be used as a diagnostic marker for oral cancer.

\section{REFERENCES}

Antic D, Keene JD (1997) Embryonic lethal abnormal visual RNA-binding proteins involved in growth, differentiation, and posttranscriptional gene expression. Am J Hum Genet 61: 273-278

Aoyagi M, Higashino F, Yasuda M, Takahashi A, Sawada Y, Totsuka Y, Kohgo T, Sano H, Kobayashi M, Shindoh M (2003) Nuclear export of the adenovirus E4orf6 protein is necessary for its ability to antagonize the apoptotic activity of the BH3-only proteins. Oncogene 22: $6919-6927$

Brennan CM, Gallouzi IE, Steitz JA (2000) Protein ligands to HuR modulate its interaction with target mRNAs in vivo. J Cell Biol 151: 1-14

Brennan CM, Steitz JA (2001) HuR and mRNA stability. Cell Mol Life Sci 58: $266-277$

Chen CY, Shyu AB (1995) AU-rich elements: characterization and importance in mRNA degradation. Trends Biochem Sci 20: $465-470$
Cytoplasmic HuR expression has been implicated in the malignancy of several types of carcinomas, such as colon, ovary, breast, salivary, uterus, larynx and prostate cancers, and has been postulated to contribute towards the cancerous malignant phenotype (Erkinheimo et al, 2003; López de Silanes et al, 2003, 2005; Denkert et al, 2004; Heinonen et al, 2005; Cho et al, 2007a, b; Niesporek et al, 2008). Our observations regarding the HuR export to the cytoplasm in oral cancer cells are similar to those previously reported in other types of carcinomas, and are thus in agreement with previous research. However, the relationship between the cytoplasmic HuR expression and the malignancy of oral carcinoma has not been established so far.

Although $\mathrm{HuR}$ is exported to the cytoplasm in a CRM1independent manner in the cells transformed by an adenovirus oncogene product (Higashino et al, 2005), the HuR export pathway in non-virus-mediated cancer cells has never been investigated. Although the possibility that $\mathrm{HuR}$ had been exported and accumulated in the cytoplasm before the LMB treatment is still remaining, the inability of LMB to inhibit the HuR export in oral cancer cells, proves that the export pathway is not CRM1dependent. The exact mechanism of HuR export in cancer cells is yet to be determined, but our results show that it occurs in a mechanism different from that of normal cells. Besides CRM1, Trn1 and Trn2 are other important nuclear export receptors that have been implicated in mediating the transport of HuR (Rebane et al, 2004). This gives rise to questions regarding the involvement of the Trn pathway in HuR export in oral cancer cells.

The AMP-activated protein kinase contributes towards the nuclear import of HuR (Wang et al, 2002), MAPK-activated protein kinase 2 increases the cytoplasmic level of HuR (Tran et al, 2003) and Protein kinase $C$ facilitates the export of HuR to the cytoplasm by phosphorylation of HuR (Doller et al, 2007, 2008). Thus, phosphorylation of HuR is important for its localisation in the cells. Moreover, recently, HuR has been shown to be phosphorylated by Cdk1 during the G2 phase, and is retained as phosphorylated HuR in the nucleus in association with the 14-3-3 protein (Kim et al, 2008). Although the status of HuR phosphorylation in cancer cells has never been elucidated, HuR phosphorylation might be abnormal, which could provide valuable insights into understanding its export mechanism.

\section{ACKNOWLEDGEMENTS}

We thank Mariko Aoyagi for her technical advice on in situ hybridisation and real-time RT-PCR. This work was supported in part by a Grant-in-Aid for Scientific Research from the Ministry of Education, Science and Culture of Japan.
Cho NP, Han HS, Soh Y, Lee KY, Son HJ (2007a) Cytoplasmic HuR overexpression is associated with increased cyclooxygenase-2 expression in laryngeal squamous cell carcinomas. Pathology 39: 545-550

Cho NP, Han HS, Soh Y, Son HJ (2007b) Overexpression of cyclooxygenase-2 correlates with cytoplasmic HuR expression in salivary mucoepidermoid carcinoma but not in pleomorphic adenoma. J Oral Pathol Med 36: 297 - 303

Denkert C, Weichert W, Pest S, Koch I, Licht D, Köbel M, Reles A, Sehouli J, Dietel M, Hauptmann S (2004) Overexpression of the embryonic-lethal abnormal vision-like protein HuR in ovarian carcinoma is a prognostic factor and is associated with increased cyclooxygenase 2 expression. Cancer Res 64: 189-195

Doller A, Akool el-S, Huwiler A, Müller R, Radeke HH, Pfeilschifter J, Eberhardt W (2008) Posttranslational modification of the AU-rich element binding protein HuR by protein kinase Cdelta elicits angiotensin 
HuR export in oral cancer cells

$\mathrm{H}$ Hasegawa et al

II-induced stabilization and nuclear export of cyclooxygenase 2 mRNA. Mol Cell Biol 28: 2608-2625

Doller A, Huwiler A, Müller R, Radeke HH, Pfeilschifter J, Eberhardt W (2007) Protein kinase C alpha-dependent phosphorylation of the mRNAstabilizing factor HuR: implications for posttranscriptional regulation of cyclooxygenase-2. Mol Biol Cell 18: 2137-2148

Erkinheimo TL, Lassus H, Sivula A, Sengupta S, Furneaux H, Hla T, Haglund C, Butzow R, Ristimäki A (2003) Cytoplasmic HuR expression correlates with poor outcome and with cyclooxygenase 2 expression in serous ovarian carcinoma. Cancer Res 63: 7591-7594

Fan XC, Steitz JA (1998) HNS, a nuclear-cytoplasmic shuttling sequence in HuR. Proc Natl Acad Sci USA 95: 15293-15298

Gallouzi IE, Brennan CM, Steitz JA (2001) Protein ligands mediate the CRM1-dependent export of HuR in response to heat shock. RNA 7: $1348-1361$

Gallouzi IE, Steitz JA (2001) Delineation of mRNA export pathways by the use of cell-permeable peptides. Science 294: 1895-1901

Heinonen M, Bono P, Narko K, Chang SH, Lundin J, Joensuu H, Furneaux H, Hla T, Haglund C, Ristimäki A (2005) Cytoplasmic HuR expression is a prognostic factor in invasive ductal breast carcinoma. Cancer Res 65: $2157-2161$

Higashino F, Aoyagi M, Takahashi A, Ishino M, Taoka M, Isobe $T$, Kobayashi M, Totsuka Y, Kohgo T, Shindoh M (2005) Adenovirus E4orf6 targets pp32/LANP to control the fate of ARE-containing mRNAs by perturbing the CRM1-dependent mechanism. J Cell Biol 170: 15-20

Jacobson A, Peltz SW (1996) Interrelationships of the pathways of mRNA decay and translation in eukaryotic cells. Annu Rev Biochem 65: 693-739

Kim HH, Abdelmohsen K, Lal A, Pullmann Jr R, Yang X, Galban S, Srikantan S, Martindale JL, Blethrow J, Shokat KM, Gorospe M (2008) Nuclear HuR accumulation through phosphorylation by Cdk1. Genes Dev 22: $1804-1815$
López de Silanes I, Fan J, Yang X, Zonderman AB, Potapova O, Pizer ES, Gorospe M (2003) Role of the RNA-binding protein HuR in colon carcinogenesis. Oncogene 22: 7146-7154

López de Silanes I, Lal A, Gorospe M (2005) HuR: post-transcriptional paths to malignancy. RNA Biol 2: $11-13$

Ma WJ, Cheng S, Campbell C, Wright A, Furneaux H (1996) Cloning and characterization of HuR, a ubiquitously expressed Elav-like protein. J Biol Chem 99: 8144-8151

Niesporek S, Kristiansen G, Thoma A, Weichert W, Noske A, Buckendahl AC, Jung K, Stephan C, Dietel M, Denkert C (2008) Expression of the ELAV-like protein HuR in human prostate carcinoma is an indicator of disease relapse and linked to COX-2 expression. Int J Oncol 32: $341-347$

Rebane A, Aab A, Steitz JA (2004) Transportins 1 and 2 are redundant nuclear import factors for hnRNP Al and HuR. RNA 10: 590-599

Shindoh M, Higashino F, Kaya M, Yasuda M, Funaoka K, Hanzawa M, Hida K, Kohgo T, Amemiya A, Yoshida K, Fujinaga K (1996) Correlated expression of matrix metalloproteinases and ets family transcription factor E1A-F in invasive oral squamous-cell-carcinoma-derived cell lines. Am J Pathol 148: $693-700$

Tran H, Maurer F, Nagamine Y (2003) Stabilization of urokinase and urokinase receptor mRNAs by $\mathrm{HuR}$ is linked to its cytoplasmic accumulation induced by activated mitogen-activated protein kinaseactivated protein kinase 2. Mol Cell Biol 23: 7177-7188

Wang W, Fan J, Yang X, Fürer-Galban S, Lopez de Silanes I, von Kobbe C, Guo J, Georas SN, Foufelle F, Hardie DG, Carling D, Gorospe M (2002) AMP-activated kinase regulates cytoplasmic HuR. Mol Cell Biol 22: $3425-3436$

Weigel S, Dobbelstein M (2000) The nuclear export signal within the E4orf6 protein of adenovirus type 5 supports virus replication and cytoplasmic accumulation of viral mRNA. J Virol 74: 764-772 